\title{
GCU
}

Glasgow Caledonian

University

University for the Common Good

\section{Agreement of the activPAL3 and activPAL for characterising posture and stepping in adults and children}

Sellers, Ceri; Dall, Philippa; Grant, Margaret; Stansfield, Ben

Published in:

Gait and Posture

DOI:

10.1016/j.gaitpost.2016.05.012

Publication date:

2016

Document Version

Author accepted manuscript

Link to publication in ResearchOnline

Citation for published version (Harvard):

Sellers, C, Dall, P, Grant, M \& Stansfield, B 2016, 'Agreement of the activPAL3 and activPAL for characterising posture and stepping in adults and children', Gait and Posture, vol. 48, pp. 209-214.

https://doi.org/10.1016/j.gaitpost.2016.05.012

\section{General rights}

Copyright and moral rights for the publications made accessible in the public portal are retained by the authors and/or other copyright owners

and it is a condition of accessing publications that users recognise and abide by the legal requirements associated with these rights.

Take down policy

If you believe that this document breaches copyright please view our takedown policy at https://edshare.gcu.ac.uk/id/eprint/5179 for details

of how to contact us. 
Title

Agreement of the activPAL3 and activPAL for characterising posture and stepping in adults and children.

\section{Authors}

Ceri Sellers ${ }^{\mathrm{a}}$, Philippa Dall ${ }^{\mathrm{a}}$, Margaret Grant ${ }^{\mathrm{a}}$, Ben Stansfield ${ }^{\mathrm{a}}$

${ }^{a}$ Institute for Applied Health Research, School of Health and Life Sciences, Glasgow Caledonian University, Cowcaddens Road, Glasgow, G4 OBA, UK.

E-mails: Ceri.Sellers@gcu.ac.uk, Philippa.Dall@gcu.ac.uk, M.Grant@gcu.ac.uk, ben.stansfield@gcu.ac.uk

Corresponding author: Ben Stansfield, Institute for Applied Health Research, School of Health and Life Sciences, Glasgow Caledonian University, Cowcaddens Road, Glasgow, G4 OBA, UK. ben.stansfield@gcu.ac.uk. Tel: 0044 (0) 1412731551.

\section{Acknowledgements (funding)}

The work completed here was funded as part of an EPSRC CASE PhD studentship award which was partly funded by PAL Technologies Ltd (Glasgow, UK), the manufacturer of the activPAL3 device. The authors would like to thank all participants for their time in taking part in this work.

\section{Highlights}

During standardised activities activPAL3 and activPAL generally have good agreement.

There are lower levels of agreement for standardised jogging activities.

For adults and young people during ADL activPAL3 records more steps than activPAL.

In young people there were differences in posture classification (sit/stand) for ADL. 


\begin{abstract}
Characterisation of physical activity and sedentary behaviour under free-living conditions is becoming increasingly important in light of growing evidence for the health implications of these behaviours. The integrity of long-term follow-up and the ability to compare outcomes between studies is critically dependent on the agreement of outcomes from successive generations of monitors. This study evaluated the agreement of the activPAL and second generation activPAL3 devices. Agreement was assessed in both adults (median 27.6y IQR 22.6) $(n=20)$ and young people (median 12.0y IQR 4.1) ( $n=8)$ during standardised and daily living (ADL) test activities. During standardised activities; sedentary duration, upright duration, stepping duration and overall number of steps were all detected within small limits of agreement $(\leq 5 \%)$. However, the activPAL characterised more steps during jogging than the activPAL3 (adults $+8.36 \%$, young people $+6.80 \%$ ). Also during ADL differences arose due to different posture characterisation in young people and lower step detection in the activPAL than the activPAL3 (adults $-20.58 \%$, young people $-11.43 \%$ ). Second-by-second posture analysis demonstrated high levels (>90\%) of agreement for all activities between monitors. However, sensitivity $(68.7 \%)$ and positive predictive value $(78.8 \%)$ for adult stepping demonstrated disagreement between monitor interpretation of movement patterns during ADL. Agreement between monitor outcomes for standardised activities provides confidence that these outcomes can be considered almost equivalent. However, for characterisation of jogging and smaller movements during ADL it is likely that significant differences between monitor outcomes will arise.
\end{abstract}

\title{
Keywords
}

Agreement; activPAL3; activPAL; Adults and Children; Posture and stepping 


\section{INTRODUCTION}

The activPAL (PAL Technologies Ltd. Glasgow, UK) family of monitors are used to characterise physical activity and sedentary behaviours. The validity and reliability of the original activPAL monitor has been studied in a variety of different populations [1-8]. A smaller, but increasing, body of evidence has been published on the newer activPAL3 model [9-12].

Agreement between the outcomes of different generations of monitors is needed to ensure compatibility between successive episodes of data collection in longitudinal studies, and to allow comparisons to be made between studies using these different monitors. It has been assumed that different generations of the same activity monitor can be used interchangeably (e.g.[13-14]); for example using validation evidence produced using the activPAL model to substantiate the use of the activPAL3 [15]. In fact, the activPAL and activPAL3 monitors have different hardware configurations and, therefore, may produce different outcomes. For example, the activPAL is a uni-axial accelerometer whereas the activPAL3 is tri-axial. As the devices use different hardware to detect acceleration, they may not produce identical acceleration signals for the same movement patterns, and are likely to use different signal analysis algorithms to determine posture and stepping. So even with the devices placed in identical positions it is possible that different outcomes may be recorded.

Only one study (Klenk et al. [16]) has reported agreement between the activPAL and activPAL3 monitors. Study participants (>65 years) carried out a sequence of standardised activities, comprising clear periods of sitting, standing and stepping, performed in a laboratory setting. Agreement between the monitors was high at a median of $96.8 \%$ (IQR 2.6\%). Whilst this first evidence of the agreement between the monitor outcomes is encouraging, the protocol used was standardised, of short duration (157 $\pm 17 \mathrm{~s})$ and did not include step count results.

Previous validation studies have demonstrated that the activPAL family of monitors characterise standardised activities very well $[1,2,12]$. However, when activities of daily living (ADL) were tested, validity was reduced. For example, Grant et al. [2] assessed the validity of the activPAL monitor in adults for both standardised and ADL activities using video observation as the criterion measure. For standardised activities, walking duration had a mean difference of $-0.7 \%$ with limits of agreement (LOA) of -4.1 to $2.7 \%$ compared to the video. However, for ADL activities the mean difference was higher with wider limits of agreement at -3.6 (LOA -59.8 to 52.6)\%. Sellers et al. [12] reported a similar effect for activPAL3 validity testing where the mean difference for stepping duration in standardised test activities was -1.42 (LOA -2.68 to -0.17 )\% for adults, whereas agreement for ADL activities was much poorer with a mean difference of -54.91 (LOA -95.18 to -14.63$) \%$. Whilst these studies were comparing activPAL and activPAL3 outputs against video observation outcomes, they 
suggest that it is important to incorporate free-choice movement into a validation protocol to gain a fuller understanding of potential outcomes.

Results from the study by Klenk et al. [16] cannot be generalised to a younger population as it is recognised that older adults may use different movement patterns [17]. Hence, further investigation is required to examine the level of agreement between the two monitors in a younger population, including a range of activities which may be carried out in the daily lives of younger people, e.g. jogging.

Therefore, the purpose of this study was to assess the agreement of the activPAL and activPAL3. It was hypothesised that there would be a high level of agreement between the monitors for both adults and young people using a protocol including standardised activities and ADL $[1,2,12]$.

\section{METHODS}

\section{Participants}

Twenty adults (18-65y) and 8 young people (6-17y) were recruited via email advertisement amongst staff and students at the authors' institution. Young people were recruited through staff members. Participants were excluded if they used mobility aids or reported movement difficulties that would affect protocol performance. Ethical approval was gained from the institutional review board. Participants/parents gave written informed consent/assent.

\section{Physical activity monitors}

The activPAL and activPAL3 monitors record posture (sedentary; upright: standing or stepping) and step count against time. Study participants wore an activPAL monitor approximately one third of the way down the front of the right thigh and secured using a PALstickies ${ }^{\mathrm{TM}}$ hydrogel pad. An activPAL3 monitor was piggybacked on top. The two devices were taped tightly together using micropore tape.

\section{Protocol}

The full test protocol has been described previously [12]. Testing sessions lasted approximately $80 \mathrm{mins}$ and included two types of test activities:

- Standardised Activities - Fourteen activities ( 8 inside, 6 outside) (Table 1) were performed by participants who stood for $15 \mathrm{~s}$ before and after performing each activity to provide a 
clear delineation in device outputs. Outdoor activities were performed using a set route on paved surfaces with kerbs and slight slopes.

- Activities of Daily Living (ADL) - Activities were based on the original activPAL validation study [2] and intended to represent activities participants might carry out in their daily lives. A total of 18 tasks (Table 1) were identified for adults [2] and 14 for young people [12]. Tasks were assigned to lists of 6 activities covering a range of postures. Each participant completed one randomly-assigned task list of 6 activities. Participants sat for 15s between activities.

Task order was randomised within sections. Testing sessions were videoed and the video record subsequently analysed to record the postures used, the timings of transitions between postures and the steps taken.

\section{Data Analysis}

Data were processed using activPAL software version 7.1.18 (2s minimum sitting and $2 \mathrm{~s}$ standing periods [18]). The events file (activPAL software output) was used to develop the analysis giving a time-stamped (nearest $10^{\text {th }}$ second) record of postural transitions and individual strides. Together with the video record, the event information provided allowed the calculation of the duration spent in different postures and the accumulation of steps (strides $\times 2$ ) for each test activity. Outcomes were aggregated giving total durations for sedentary and upright postures, as well as breaking upright duration into standing and stepping durations. Step count for activities involving walking and jogging was determined. Totals were calculated separately for the two types of test activities; standardised and ADL. Modified Bland and Altman plots $[19,20]$ were used to analyse outcomes with a-priori limit of acceptability for limits of agreement of $\leq \pm 5 \%$.

An analysis of the continuous record of posture and steps during the ADL testing was undertaken to examine second-by-second agreement between monitors using the activPAL as the reference measure. Agreement, sensitivity and positive predictive value (PPV) were calculated as follows [21]:

$$
\begin{aligned}
& \% \text { agreement }=\frac{\text { Number of seconds where activPAL3 posture }=\text { activPAL posture }}{\text { Number of seconds }} \times 100 \\
& \text { Sensitivity }=\frac{\text { No. of seconds where activPAL3 posture }=\text { A and activPAL posture }=\mathrm{A}}{\text { Number of seconds where activPAL posture }=\mathrm{A}} \times 100 \\
& \mathrm{PPV}=\frac{\text { Number of seconds where activPAL3 posture }=\text { A and activPAL posture }=\mathrm{A}}{\text { Number of seconds where activPAL3 }=\mathrm{A}} \times 100
\end{aligned}
$$


Agreement indicates percentage of time the activPAL3 and activPAL monitors agree. Sensitivity indicates the percentage of time that the activity (e.g. sitting) measured by the activPAL monitor is also detected as the same activity by activPAL3. PPV gives the percentage of time that the activity measured by activPAL3 matches activPAL.

\section{RESULTS}

As previously reported [12], twenty adults (9M,11F), median age 27.6y (IQR 22.6) and mean body mass index (BMI) $24.6 \pm 3.2 \mathrm{~kg} / \mathrm{m}^{2}$ and 8 young people (2M/6F) median age $12.0 \mathrm{y}$ (IQR 4.1) participated. All young people were normal weight according to the 1990 British BMI reference curves [22]. Walking speeds ranged between $0.98-2.24 \mathrm{~ms}^{-1}$ for adults and $0.92-2.06 \mathrm{~ms}^{-1}$ for young people, with jogging speeds between $1.4-4.1 \mathrm{~ms}^{-1}$ for adults and $2.1-3.3 \mathrm{~ms}^{-1}$ for young people. The mean test duration of standardised activities excluding breaks was $26 \mathrm{mins}$ for the adults and $24 \mathrm{mins}$ for the young people. The mean ADL test duration for both adults and young people was $11 \mathrm{mins}$. One young person did not perform the treadmill jogging activity. The two youngest children carried out 'external' activities indoors (55m walking; $23 \mathrm{~m}$ jogging).

For standardised activities, agreement for sedentary and upright durations was $< \pm 1 \%$ for both adults and young people, with LOA well within the a-priori acceptable limit of $\pm 5 \%$ (Table 2) (Modified Bland and Altman plots - supplementary Figures S1-S2). Agreement for step detection was again within the $5 \%$ limit (LOA: stepping duration $< \pm 2 \%$, step count $< \pm 4 \%$ ). However, agreement for jogging steps was poorer with the activPAL3 recording $8.36 \%$ fewer steps for adults and $6.80 \%$ fewer for young people than the activPAL (Table 2).

For $A D L$ activities, sedentary duration and upright duration limits of agreement were again within the $5 \%$ limit for adults $(< \pm 1 \%)$, in contrast with young people where on average activPAL3 recorded 13.72\% less sedentary duration and $7.52 \%$ more upright duration than activPAL. The activPAL3 monitor recorded more steps in both adults and young people during ADL activities $(20.58 \%$ and $11.43 \%$ respectively).

Analysis of recorded posture on a second-by-second basis was carried out for classification of posture into upright and sedentary, and for classification into sedentary, standing and stepping. Agreement between activPAL and activPAL3 monitors was $>90 \%$ for adults and young people for both standardised and ADL activities (Table 3). Sensitivity and PPV for upright/sedentary posture categorisation ranged between $91.5-100 \%$ for all activities. Results for posture categorisation into sedentary/standing/stepping were lower; Sensitivity and PPV were $>90 \%$ for all standardised 
activities except young people's standing, which was $85.4 \%$. Sensitivity and PPV values were lower for ADL activities for both adults and young people, the lowest being for adults' stepping duration (PPV 68.7\%).

\section{DISCUSSION}

The activPAL family of monitors has been widely used to record physical activity and sedentary behaviour. The ability to accurately compare or combine results from studies using different models of the activPAL family of monitors relies on a high level of agreement between reported outcomes. Hardware changes between the activPAL and activPAL3 generations of devices may have led to differences in outcomes. Previous examination of agreement between the monitors in older adults [16] was performed using a standardised protocol which demonstrated high levels of agreement between monitors on a second-by-second basis (highest disagreement for standing; median 95.0 $($ IQR 4.0$) \%)$. The current study is the first to investigate the agreement of the activPAL and activPAL3 activity monitors for both posture and step count, using a range of both standardised activities and activities of daily living in both adults and children. The comparison of outputs between monitors was, therefore, tested not only in clearly defined postures with stepping, but also in self-selected postures and movement patterns associated with normal daily activities.

For standardised activities, the two monitors characterised activities in a very similar way with agreement within the a-priori limits set of $\pm 5 \%$, except standing and jogging (Table 2). Both monitors tended to record a small amount of time at the start or end of a stepping bout as standing for some participants. It is possible that initiating and terminating steps are taken at a slower speed, or are of shorter step length. This might have led to under-detection, as it has been demonstrated that slower stepping may not be detected [10]. The activPAL3 monitor was more accurate in this respect, hence the mean difference for standing was approximately $-2 \%$, but the lower LOA was slightly outside the $-5 \%$ limit (Adults: $-7.41 \%$; Young People: $-6.12 \%$ ). Klenk et al. [16] also reported a higher amount of stepping duration detection by the activPAL3 than the activPAL for standardised activities for older adults. An increase of $2 \mathrm{~s}$ within a total stepping duration of $35-75 \mathrm{~s}$ was reported, giving a percentage increase of $2.5-5.5 \%$. The slightly higher difference found by Klenk et al. may have resulted from the high number of stepping bouts contained within the relatively short test protocol. The detection of jogging steps was lower for activPAL3 than activPAL in both adults and young people. This finding seems to agree with previous evidence that the activPAL monitor [23] over-estimated steps for treadmill jogging in adults by 10-15\%, while activPAL3 [12] (matched data 
to current report) undercounted jogging steps in adults and young people. However, wide limits of agreement in the current study for jogging indicate agreement between monitors was variable, with high levels of agreement for some individuals and large differences for others (Figure 1). When results for each monitor were compared with video observation (Supplementary Figure S3), it was observed that both monitors tended to undercount jogging steps, with an increasing likelihood of missing steps at higher cadences. This may be due to mechanical differences in running gait at higher cadences, such as a reduced vertical displacement of the centre of mass [24] with subsequent change to thigh kinematics.

While agreement between the monitors was close for most young people, activPAL3 recorded a much lower sitting duration than activPAL for two participants who were perching on the edge of their seats resulting in wide LOA (-58.41 to $30,98 \%$, Table 2$)$. Without these outliers, the mean difference was -1.45 (LOA -6.05 to 3.15 )\% for sitting duration and -4.42 (LOA -9.46 to 0.62 )\% for standing. It has previously been reported that perching can cause misclassification of sitting as standing (activPAL [25], activPAL3 [12]). The current study results suggest that the two monitors may be affected differently by this phenomenon. Klenk at al. [16] also reported three participants with sitting duration at least $20 \%$ lower for activPAL3 than activPAL.

The current study demonstrated only a small difference between activPAL and activPAL3 stepping duration for standardised activities (0.31 (LOA -0.53 to 1.16 )\% for adults; 0.31 (LOA -0.14 to 0.76 )\% for young people). However, activPAL3 detected a substantially higher stepping duration than activPAL for ADL activities: 18.68 (LOA -17.92 to 55.28 )\% for adults and 7.14 (LOA -2.05 to 16.33)\% for young people. Stepping behaviour during ADL test activities typically consisted of purposeful periods of stepping (e.g. walking from one side of the room to the other), interspersed with periods of very small positional steps used to precisely locate the body closer to a task (e.g. small stepping movements at a kitchen sink when washing up) and slow stepping used when walking while carrying out a task (such as hanging out washing on a clothes drier). Some of the ADL tasks for young people also included short bouts of faster stepping (e.g. playing indoor basketball). As both monitors have been found to detect purposeful stepping accurately $[1,2,12]$, detection of a higher number of steps during ADL activities suggests the activPAL3 may show a greater sensitivity to smaller positional stepping movements or slower stepping. However, the second-by-second analysis showed that both sensitivity and PPV were relatively low for stepping in ADL activities for adults, indicating that there were periods of stepping reported by the activPAL monitor that were not detected as stepping by the activPAL3, and vice versa (Table 3). This was also the case, but to a lesser extent, for the young people. Figure 2 shows an example of this where the activPAL3 monitor recorded stepping activity at the start of an ADL activity for adult participant P1, but the activPAL monitor did not. Both 
monitors picked up stepping between seconds 20-35, but the activPAL3 failed to pick up the next two short bouts of steps. It was discussed earlier that the activPAL monitor tended to miss the start or end of a stepping bout more than the activPAL3 monitor. Differences between the monitors in detection of slow-stepping may also be having an effect, however there is limited evidence on how well the monitors measure stepping at the very low cadences used in this type of activity $[4,12]$. One study [10] reported that the activPAL3 records $90 \%$ of steps at cadences $\geq 69$ steps/minute with an increasing probability of missed steps at lower cadences. As a typical ADL activity for one participant had a median cadence of 85.7 (IQR 36.7) steps/minute, it seems possible that slowstepping occurring during some ADL activities would not be accurately recorded by the activPAL3. A combination of these factors, in addition to individual specific movement patterns, perhaps relating to step length or cadence combinations used when slow-stepping, may have influenced the relative performance of the activPAL and activPAL3 during ADL test activities. Further investigation into the agreement of the two monitors at very slow cadences may be warranted.

For the current analysis it was important that activities could be observed so that participants' actual physical activity behaviour could be recorded. An extension of this analysis would have been to examine agreement between the monitors during unobserved free-living activity across longer time periods. However, the exact activity being undertaken during these time periods would not have been known, making it difficult to identify the movements causing disagreements between the monitors, and identification of the monitor which was recording the correct posture or number of steps.

Second-by-second between monitor agreement for standardised and ADL activities was $>91 \%$ for both adults and young people (Table 3). Agreement between monitors for adults for standardised activities for separation of time into stepping/standing/sedentary was $99.0 \%$, very similar to Klenk et al. [16]. The second-by-second analysis provides additional evidence that differences arise in the monitor outputs for activities that are not of a standardised nature, again emphasising the need to assess monitor agreement across a range of activities.

\section{SUMMARY}

The use of a protocol including both standardised activities and ADL has highlighted the difference in outcomes of the devices under these two conditions. In general for standardised activities there was good agreement between monitor outcomes. The exception to this was that the activPAL3 detected lower numbers of jogging steps than the activPAL. For ADL there was generally considerably lower agreement between monitor outcomes than for standardised activities. For ADL in adults there was 
higher step detection by the activPAL3 than the activPAL. For the young people, in addition to similar differences in step detection there were differences in detected posture, with sedentary and standing duration being swapped due to perching on the edge of seats. The extent to which the levels of agreement reported here will impact on real life day-to-day activity characterisation would depend on the particular activities being undertaken. However, for the performance of simple movement patterns agreement is in general good, but caution must be exercised if the study population perform large volumes of complex or non-standard movements.

\section{REFERENCES}

[1] Ryan CG, Grant PM, Tigbe WW, Granat MH. The validity and reliability of a novel activity monitor as a measure of walking. Br J Sports Med 2006; 40: 779-84.

[2] Grant PM, Ryan CG, Tigbe WW, Granat, MH. The validation of a novel activity monitor in the measurement of posture and motion during everyday activities. Br J Sports Med 2006; 40: 992-7. [3] Ryan CG, Grant PM, Gray H, Newton M, Granat MH. Measuring postural physical activity in people with chronic low back pain. J Back Musculoskelet Rehabil 2008; 21(1): 43-50.

[4] Grant PM, Dall PM, Mitchell SL, Granat MH. Activity-monitor accuracy in measuring step number and cadence in community-dwelling older adults. J Aging Phys Act 2008; 16(2): 201-14.

[5] Davies G, Reilly J, McGowan A, Dall P, Granat M, Paton J. Validity, practical utility, and reliability of the activPAL in preschool children. Med Sci Sports Exerc 2012; 44(4): 761-8.

[6] Aminian S, Hinckson EA. Examining the validity of the activPAL monitor in measuring posture and ambulatory movement in children. Int J Behav Nutr Phys Act 2012; 9: 119.

[7] Harrington D. Objective assessment and modification of physical activity and health indices in adolescent females [dissertation]. Limerick, Ireland: University of Limerick; 2010.

[8] Dowd KP, Harrington DM, Donnelly AE. Criterion and concurrent validity of the activPAL ${ }^{\mathrm{TM}}$ professional physical activity monitor in adolescent females. PLoS One 2012; 7(10): e47633.

[9] Berendsen BAJ, Hendriks MRC, Meijer K, Plasqui G, Schaper NC, Savelberg HHCM. Which activity monitor to use? Validity, reproducibility and user friendliness of three activity monitors. BMC Public Health 2014; 14: 749.

[10] Stansfield B, Hajarnis M, Sudarshan R. Characteristics of very slow stepping in healthy adults and validity of the activPAL3 ${ }^{\mathrm{TM}}$ activity monitor in detecting these. Med Eng Phys 2015; 37: $42-7$. 
[11] Ryde GC, Gilson ND, Suppini A, Brown WJ. Validation of a novel, objective measure of occupational sitting. J Occup Health 2012; 54: 383-6.

[12] Sellers C, Dall P, Grant M, Stansfield B. Validity and reliability of the activPAL3 for measuring posture and stepping in adults and young people. Gait Posture 2016; 43: 42-7.

[13] Aguilar-Farías N, Brown W, Olds T, Peeters G. Validity of self-report methods for measuring sedentary behaviour in older adults. J Sci Med Sport 2015; 18(6): 662-6.

[14] Varela-Mato V, Yates T, Stensel D, Biddle S, Clemes S. Time spent sitting during and outside working hours in bus drivers: A pilot study. Prev Med Rep 2016; 3: 36-9.

[15] Gorman E, Ashe MC, Dunstan DW, Hanson HM, Madden K, Winkler EAH et al. Does an 'activitypermissive' workplace change office workers' sitting and activity time? PLoS One 2013; 8(10): e76723.

[16] Klenk J. Büchele G, Lindemann U, Kaufmann S, Peter R, Laszlo R et al. Concurrent validity of activPAL and activPAL3 accelerometers in older adults. J Aging Phys Act (in press) DOI: http://dx.doi.org/10.1123/japa.2015-0178.

[17] Godfrey A, Conway R, Meagher D, ÓLaighin G. Direct measurement of human movement by accelerometry. Med Eng Phys 2008; 30: 1364-86.

[18] Alghaeed Z, Reilly JJ, Chastin SFM, Martin A, Davies G, Paton JY. The influence of minimum sitting period of the activPAL ${ }^{\mathrm{TM}}$ on the measurement of breaks in sitting in young children. PLoS One 2013; 8(8): e71854.

[19] Bland JM, Altman DG. Statistical methods for assessing agreement between two methods of clinical measurement. Lancet 1986; i: 307-10.

[20] Linnet K, Bruunshuus I. HPLC with enzymatic detection as a candidate reference method for serum creatinine. Clin Chem 1991; 37: 1669-75.

[21] Bussmann HBJ, Reuvekamp PJ, Veltink PH, Martens WLJ, Sta HJ. Validity and reliability of measurements obtained with an "activity monitor" in people with and without a transtibial amputation. Phys Ther 1998; 78(9): 989-98.

[22] Cole TJ, Freeman JV, Preece MA. Body mass index reference curves for the UK, 1990. Arch Dis Child 1995; 73: 25-9.

[23] Dahlgren G, Carlsson D, Moorhead A, Häger-Ross C, McDonough SM. Test-retest reliability of step counts with the ActivPAL device in common daily activities. Gait Posture 2010; 32: 386-90. 
[24] Heiderscheit BC, Chumanov ES, Michalski MP, Wille CM, Ryan MB. Effects of step rate manipulation on joint mechanics during running. Med Sci Sports Exerc 2011; 43(2): 296-302.

[25] Tang KT, Richardson AM, Maxwell D, Spence WD, Stansfield BW. Evaluation of an activity monitor for the objective measurement of free-living physical activity in children with cerebral palsy. Arch Phys Med Rehabil 2013; 94: 2549-58. 
Table 1 Test activities for adults and young people.

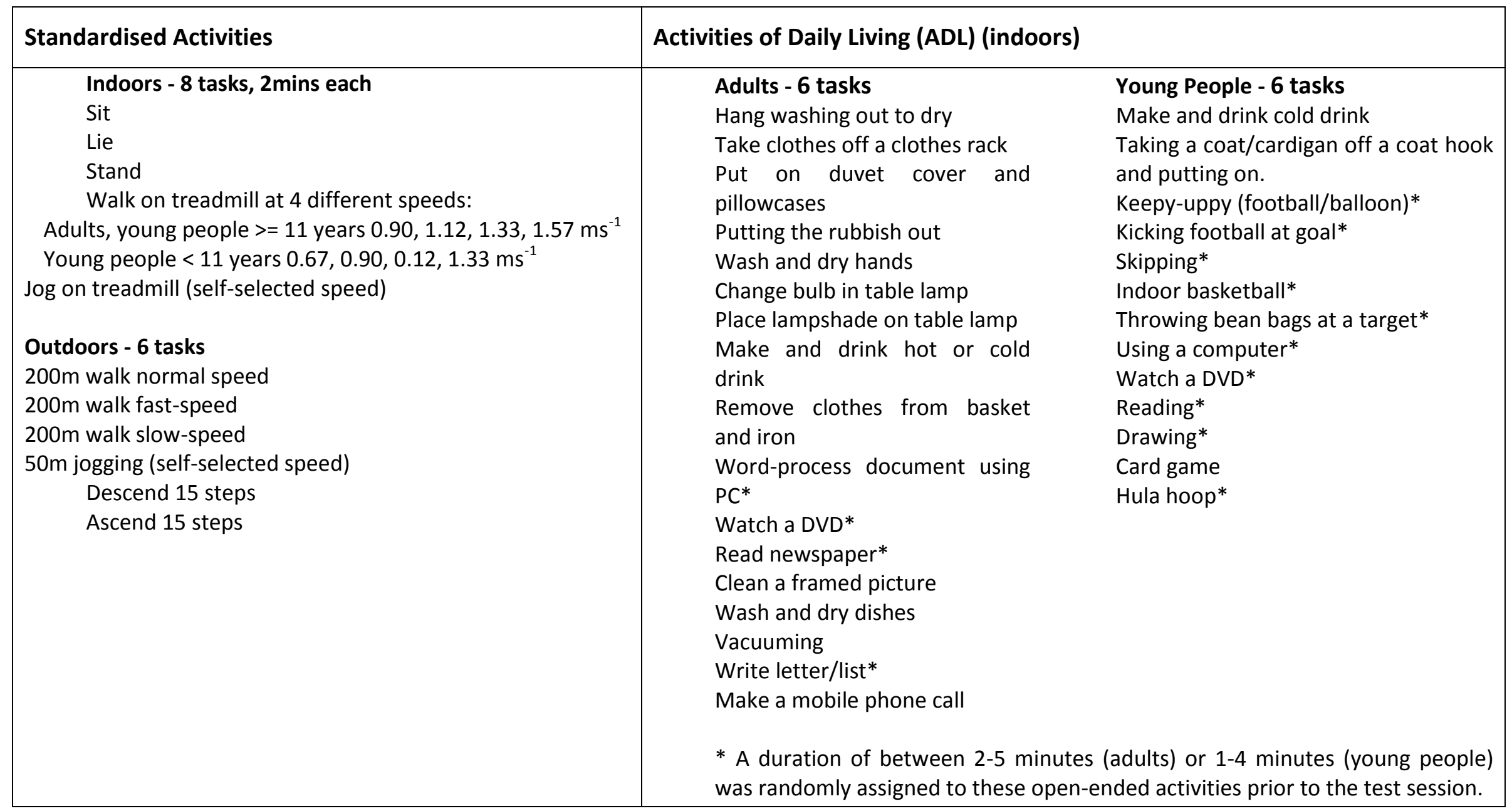


Table 2 Bland and Altman Percentage Mean Differences: activPAL and activPAL3 (a positive value indicates activPAL3 was higher than activPAL).

\begin{tabular}{|c|c|c|c|c|}
\hline \multirow{3}{*}{ Measure } & \multicolumn{4}{|c|}{ Percentage Mean Difference (LLOA, ULOA) (\%) } \\
\hline & \multicolumn{2}{|c|}{ Standardised Activities } & \multicolumn{2}{|c|}{ Activities of Daily Living (ADL) } \\
\hline & Adults & Young People & Adults & Young People \\
\hline \multicolumn{5}{|l|}{ Duration } \\
\hline Sedentary & $-0.07(-0.25,0.12)^{* *}$ & $-0.08(-0.23,0.07)^{* *}$ & $-0.18(-0.41,0.05)^{* *}$ & $-13.72(-58.41,30.98)$ \\
\hline Upright & $0.07(-0.29,0.43)^{* *}$ & $0.04(-0.66,0.75)^{* *}$ & $0.40(-0.01,0.82)^{* *}$ & $7.52(-17.83,32.88)$ \\
\hline Standing & $-1.97(-7.41,3.46)$ & $-2.08(-6.12,1.95)$ & $-3.38(-11.47,4.72)$ & $8.30(-40.86,57.45)$ \\
\hline Stepping & $0.31(-0.53,1.16)^{* *}$ & $0.31(-0.14,0.76)^{* *}$ & $18.68(-17.92,55.28)$ & $7.14(-2.05,16.33)$ \\
\hline \multicolumn{5}{|l|}{ Step Count } \\
\hline All activities & $-1.36(-3.85,1.13)^{* *}$ & $-1.60(-3.94,0.74)^{* *}$ & $20.58(-11.27,52.42)$ & $11.43(-2.41,25.27)$ \\
\hline All activities excluding jogging & $0.06(-1.03,1.16)^{* *}$ & $-0.04(-1.01,0.93)^{* *}$ & & \\
\hline Jogging activities only & $-8.36(-22.48,5.76)$ & $-6.80(-32.41,18.82)$ & & \\
\hline
\end{tabular}

** met the criteria of $\pm 5 \%$ limits of agreement LLOA Lower limit of agreement ULOA Upper limit of agreement 
Table 3 Second-by-second posture agreement, sensitivity and positive predictive value (PPV): activPAL and activPAL3.

\begin{tabular}{|c|c|c|c|c|c|c|c|c|c|c|c|c|}
\hline \multirow[b]{3}{*}{ Measure } & \multicolumn{6}{|c|}{ Standardised Activities } & \multicolumn{6}{|c|}{ Activities of Daily Living (ADL) } \\
\hline & \multicolumn{3}{|c|}{ Adults } & \multicolumn{3}{|c|}{ Young People } & \multicolumn{3}{|c|}{ Adults } & \multicolumn{3}{|c|}{ Young People } \\
\hline & 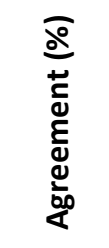 & 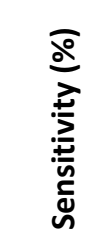 & $\frac{\grave{a}}{a}$ & 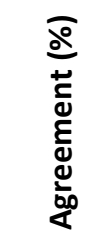 & 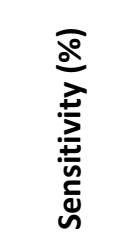 & $\frac{\grave{a}}{a}$ & 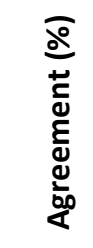 & 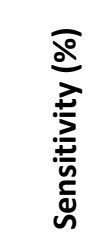 & $\frac{\grave{9}}{a}$ & 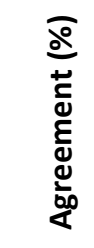 & 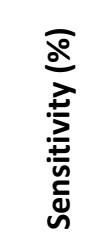 & $\frac{\grave{2}}{a}$ \\
\hline $\begin{array}{l}\text { Upright, Sedentary } \\
\text { Sedentary } \\
\text { Upright }\end{array}$ & 99.9 & $\begin{array}{l}99.9 \\
99.9\end{array}$ & $\begin{array}{c}99.7 \\
100.0 \\
\end{array}$ & 99.7 & $\begin{array}{c}100.0 \\
99.6\end{array}$ & $\begin{array}{c}98.3 \\
100.0 \\
\end{array}$ & 98.9 & $\begin{array}{l}98.7 \\
99.0\end{array}$ & $\begin{array}{l}99.0 \\
98.7\end{array}$ & 95.2 & $\begin{array}{l}91.5 \\
99.1 \\
\end{array}$ & $\begin{array}{l}99.5 \\
92.2 \\
\end{array}$ \\
\hline $\begin{array}{l}\text { Stepping, Standing, } \\
\text { Sedentary } \\
\text { Standing } \\
\text { Stepping }\end{array}$ & 99.0 & $\begin{array}{l}94.9 \\
99.5\end{array}$ & $\begin{array}{l}97.0 \\
99.1\end{array}$ & 97.7 & $\begin{array}{l}85.4 \\
99.6\end{array}$ & $\begin{array}{l}97.8 \\
97.4\end{array}$ & 94.6 & $\begin{array}{l}91.3 \\
78.8\end{array}$ & $\begin{array}{l}94.1 \\
68.7\end{array}$ & 91.4 & $\begin{array}{l}87.4 \\
93.4\end{array}$ & $\begin{array}{l}81.9 \\
86.7\end{array}$ \\
\hline
\end{tabular}




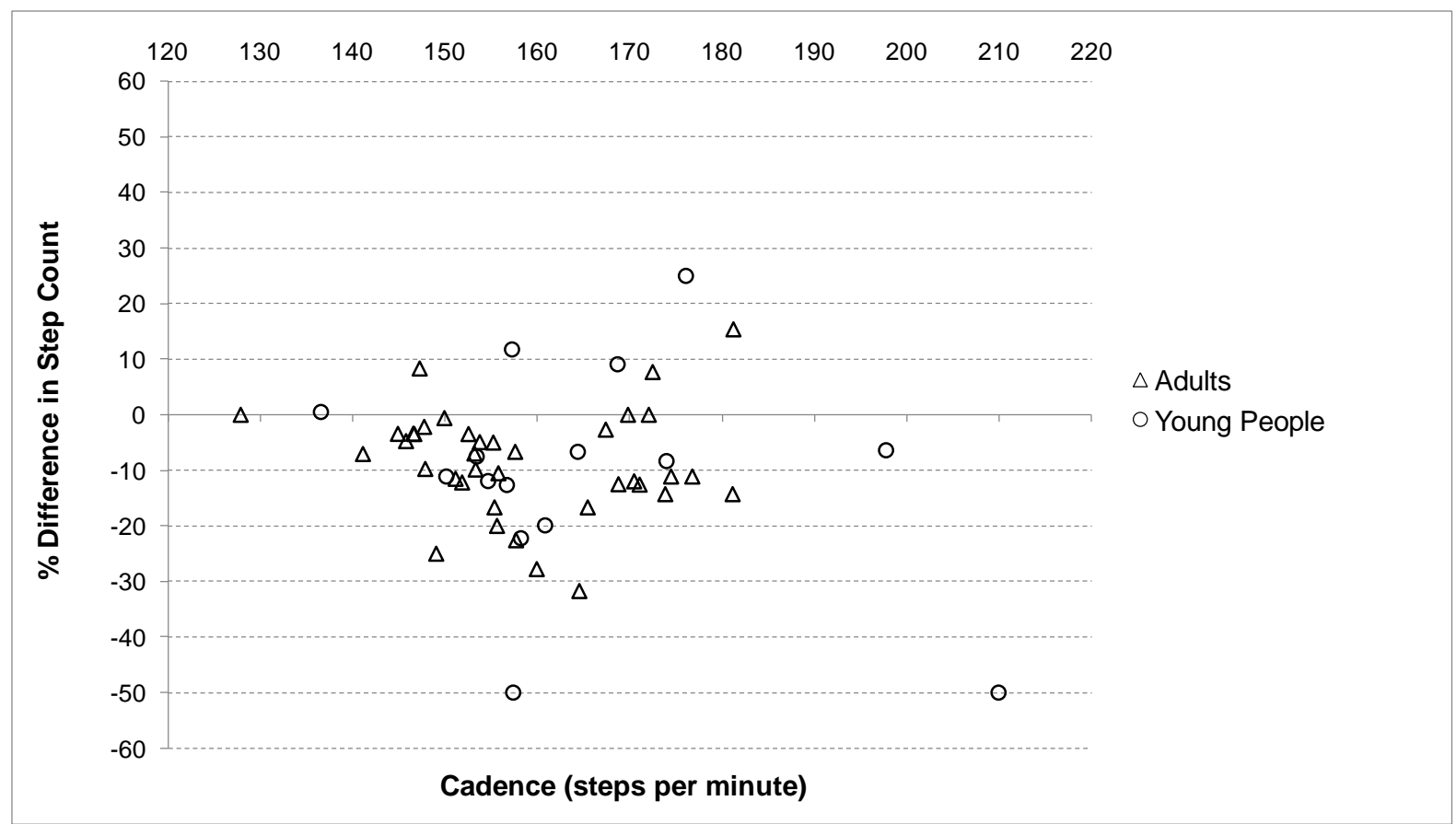

Figure 1 Percentage difference in step count between activPAL and activPAL3 for adults and young people jogging at self-selected speeds (a positive value indicates activPAL3 was higher than activPAL). The $x$ axis shows the cadence measured by video observation. 


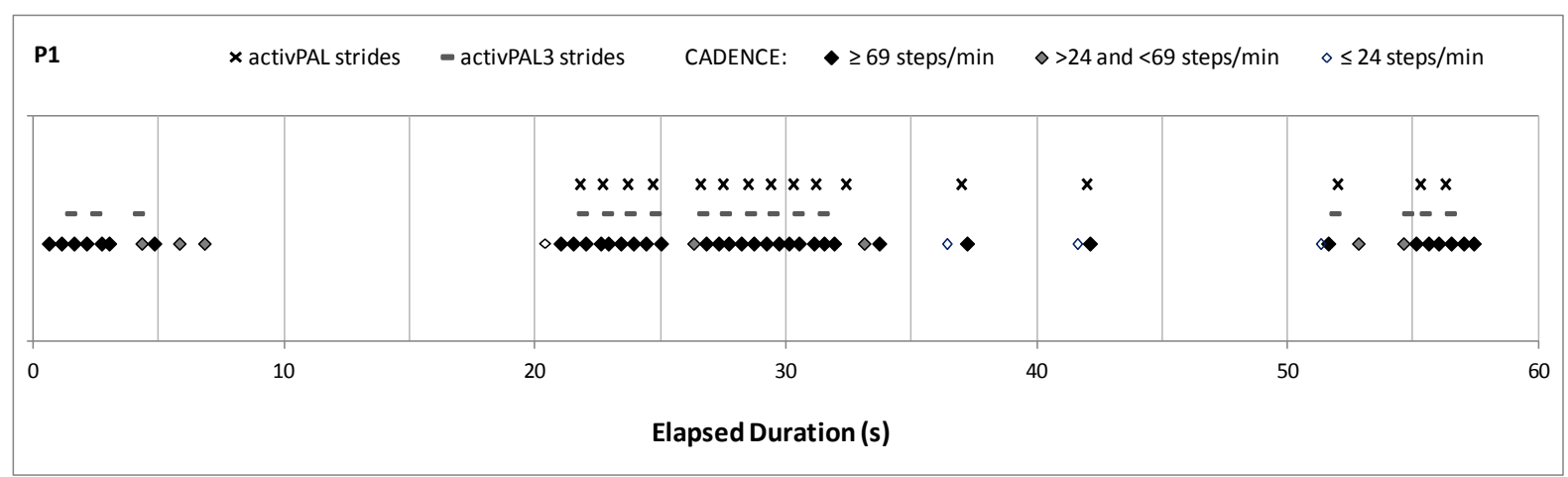

Figure 2 Plot of steps recorded by activPAL and activPAL3 monitors against steps taken according to video observation for the ADL activity "Taking the Rubbish Out" by adult participant P1 (Line 1: activPAL strides recorded, where 1 stride = 2 steps; Line 2: activPAL3 strides recorded; Line 3: Steps observed on Video Record, with shading according to cadence of step). 